International Journal of Engineering \&Technology, 8(4) (2019) $417-423$
International Journal of Engineering \& Technology
SPC
Website: www.sciencepubco.com/index.php/IJET
Research paper

\title{
Moment method approach to analyze waveguide array radiator with crossed dipole as a near field sensor
}

\author{
Suma. M ${ }^{1 *}$, Goutham. M. A. ${ }^{1}$, Paramesha ${ }^{2}$ \\ ${ }^{1}$ Department of Electronics and Communication Engineering Adichunchanagiri Institute of Technology, \\ Chikkamagaluru, 577102, Karnataka \\ ${ }^{2}$ Department of Electronics and Communication Engineering Government Engineering College, Hassan 573202, Karnataka \\ *Corresponding author E-mail: suma.aug13@gmail.com
}

\begin{abstract}
Moment method approach is used to estimate the error encountered by the sensor in the near field and probe measures co-pole and crosspole voltages at the same time. Conversely, due to several reflections between the waveguide and dipole sensor, near field values are changed. Calculations are done to analyze the absolute sampled co-pole voltage pattern and relative sampled electric field (without probe) pattern in the scan plane, and inaccuracy induced by the sensor is determined. The waveguide reflection coefficient, radiation admittance and susceptance with measuring sensor are reformed with respect to the equivalent values in the absence of the sensor in the near field.
\end{abstract}

Keywords: Admittance; Moment Method; Reflection Coefficient; Susceptance; Waveguide.

\section{Introduction}

The problem of modeling the error induced by the near-field sensor and the impedance seen by the open-ended waveguide is extremely important in order to estimate the electromagnetic susceptibility of the waveguide. Thin, finite length, perfectly conducting crossed dipole alone measures in the near field values, this introduces error due to the multiple reflection between the radiator and dipole, scattering properties of the dipole and mutual coupling effects of the poles of the dipole. As far field measurement is not practicable in most of the cases, these near field values are transformed into a far field pattern. Also for enhancing the accuracy of the theoretically predicted result, it is necessary to evaluate the field at the radiating aperture instead of assuming those fields, which was done by many researchers [1], [2]. Therefore, it is necessary to determine the radiated field at any point in the free space accurately.

The crossed-dipole is an important probe, since it measures both co-pole and cross-pole components simultaneously. The earlier researchers [3], [4] neglected effects of multiple reflections between the radiator and probe. However, the mutual coupling effect between poles of the dipole must be determined, since the cross-polarized components are generated in the near-fields. This paper has made an effort to fill in these gaps in the existing state of knowledge.

The situation addressed above requires an analysis for an open ended waveguide radiator aperture in the transmitting mode. The algorithm once developed can be used for finding out those for different broadband and narrow-band configurations of sensors and will make the sensor calibration possible without going into the rigour and expense of experimentation.

The rectangular opening in an unbounded ground plane fed by a waveguide being considered as a transmitter is excited in the dominant $\mathrm{TE}_{10}$ mode. The waveguide opening electric field and the induced current on dipole is described by weighted sinusoidal global basis functions and Dirac delta functions respectively. The boundary conditions at the waveguide opening and on the axis of sensors are enforced by taking the properties of reciprocal connection and multiple reflections between transmitter and probe. The waveguide radiator reflection coefficient and probe voltage across $50 \mathrm{ohms}$ terminating impedance are determined using the known coefficients of the entire domain and pulse basis functions respectively. Because of the multiple reflections between the radiator and probe, radiator reflection coefficient and near-field values are changed.

Many authors used different approaches such as a transverse operator method, variational, correlation matrix and integral equation method [5] - [7], while others [8] - [10] have used moment method technique to explain the waveguide problems. An electromagnetic wave in an open space incident on a thin, finite, perfectly conducting wire was analyzed by [9]. Moment method analysis of waveguide radiator with dipole and crossed dipole as the near field sensor are given by the authors [9] and [10].

\section{Theoretical formulation}

The crossed dipole in the near field of an open ended two waveguide radiator array of opening width and breadth of $2 \mathrm{a} \times 2 \mathrm{~b}$ in an unbounded base plane is displayed as in Figure 1. 


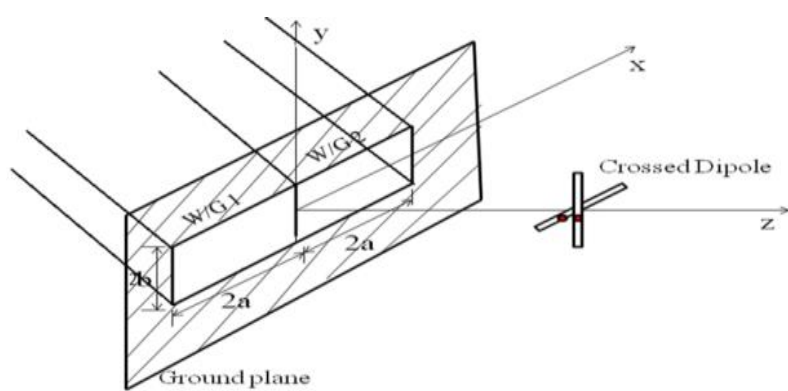

Fig. 1: The Rectangular Waveguide Array Feeding the Transmitter Is Excited in the Dominant $\mathrm{TE}_{10}$ Mode.

The incident magnetic field at the transmitter openings 1 and 2 for the dominant mode is respectively given as [8]:

$$
\begin{aligned}
& H_{x}^{i n c 1}=-Y_{01} \cos \left(\frac{\pi x}{2 a}\right) e^{-j \beta z}, \\
& H_{x}^{\text {inc2 }}=-Y_{02} \cos \left(\frac{\pi x}{2 a}\right) e^{-j \beta z},
\end{aligned}
$$

Where $\mathrm{Y}_{01}, \mathrm{Y}_{02}=$ constants at the transmitter openings 1 and 2 respectively.

The induced current at the dipole is designated by [10]:

$$
\begin{aligned}
& \mathrm{I}_{\mathrm{y}}=\hat{\mathrm{u}}_{\mathrm{y}} \sum_{\mathrm{p}=1}^{\mathrm{M}} \mathrm{I}_{\mathrm{yp}} \mathrm{i}_{\mathrm{yp}}, \\
& \mathrm{I}_{\mathrm{x}}=\hat{\mathrm{u}}_{\mathrm{x}} \sum_{\mathrm{p}=1}^{\mathrm{M}} \mathrm{I}_{\mathrm{xp}} \mathrm{i}_{\mathrm{xp}},
\end{aligned}
$$

where $\hat{\mathrm{u}}_{\mathrm{x}}, \widehat{\mathrm{u}}_{\mathrm{y}}=$ unit vectors for dipole, $\mathrm{M}=$ number of unknown basis functions, $\mathrm{I}_{\mathrm{xp}}, \mathrm{I}_{\mathrm{yp}}=$ co-efficient of the pulse basis functions for dipole, $\mathrm{i}_{\mathrm{xp}}, \mathrm{i}_{\mathrm{yp}}=$ pulse basis function in $\mathrm{x}$ and $\mathrm{y}$-direction for dipole and defined as [10]:

$\mathrm{i}_{\mathrm{yp}}=\left\{\begin{array}{c}1 \mathrm{y}_{\mathrm{p}-1} \leq \mathrm{y} \leq \mathrm{y}_{\mathrm{p}} \\ 0 \text { elsewhere }\end{array}\right.$,

$\mathrm{i}_{\mathrm{xp}}=\left\{\begin{array}{c}1 \mathrm{x}_{\mathrm{p}-1} \leq \mathrm{x} \leq \mathrm{x}_{\mathrm{p}} \\ 0 \text { elsewhere }\end{array}\right.$,

The transmitted magnetic field at the plane of the waveguide opening 1 and 2 are calculated using the plane-wave spectrum method and given by [8]:

$$
\begin{aligned}
& \mathrm{H}_{\mathrm{X}}^{\mathrm{ext} 1}=-\frac{\mathrm{ab}}{\pi^{2} \mathrm{k} \eta} \sum_{\mathrm{p}=1}^{\mathrm{M}} \mathrm{E}_{\mathrm{p}}^{1} \int_{-\infty}^{\infty} \int_{-\infty}^{\infty} \frac{\mathrm{k}^{2}-\mathrm{k}_{\mathrm{x}}^{2}}{\left(\mathrm{k}^{2}-\mathrm{k}_{\mathrm{x}}^{2}-\mathrm{k}_{\mathrm{y}}^{2}\right)^{\frac{1}{2}}} \\
& \operatorname{sinc}\left(k_{y} b\right) \frac{\left\{\begin{array}{l}
j \sin \left(k_{x} a\right) p \text { even } \\
\cos \left(k_{x} a\right) p \text { odd }
\end{array}\right\}}{\frac{p \pi}{2}\left\{1-\left(\frac{2 \mathrm{ak}_{\mathrm{x}}}{\mathrm{p \pi}}\right)^{2}\right\}} \\
& e^{j\left(k_{x} x+k_{y} y\right)} d k_{x} d k_{y} \text {, } \\
& \mathrm{H}_{\mathrm{X}}^{\mathrm{ext} 2}=-\frac{\mathrm{ab}}{\pi^{2} \mathrm{k} \eta} \sum_{\mathrm{p}=1}^{\mathrm{M}} \mathrm{E}_{\mathrm{p}}^{2} \int_{-\infty}^{\infty} \int_{-\infty}^{\infty} \frac{\mathrm{k}^{2}-\mathrm{k}_{\mathrm{x}}^{2}}{\left(\mathrm{k}^{2}-\mathrm{k}_{\mathrm{x}}^{2}-\mathrm{k}_{\mathrm{y}}^{2}\right)^{\frac{1}{2}}} \\
& \operatorname{sinc}\left(\mathrm{k}_{\mathrm{y}} \mathrm{b}\right) \frac{\left\{\begin{array}{l}
j \sin \left(\mathrm{k}_{\mathrm{x}} \mathrm{a}\right) \mathrm{p} \text { even } \\
\cos \left(\mathrm{k}_{\mathrm{x}} \mathrm{a}\right) \mathrm{p} \text { odd }
\end{array}\right\}}{\frac{\mathrm{p} \pi}{2}\left\{1-\left(\frac{2 \mathrm{ak}_{\mathrm{x}}}{\mathrm{p} \pi}\right)^{2}\right\}} \\
& e^{j\left(k_{x} x+k_{y} y\right)} d k_{x} d k_{y} \text {, }
\end{aligned}
$$

Where $\mathrm{E}_{\mathrm{p}}^{1}, \mathrm{E}_{\mathrm{p}}^{2}=$ basis functions at the transmitter apertures, $\mathrm{k}=$ Free space wave number, $\mathrm{k}_{\mathrm{x}}, \mathrm{k}_{\mathrm{y}}, \mathrm{k}_{\mathrm{z}}=$ wave number in $\mathrm{x}, \mathrm{y}$ and $\mathrm{z}$ directions respectively.

The emitted electric field at the near field sensor is calculated by [8]:

$E_{X}=0$, (no field exists due to TE10 mode excitation)

$\mathrm{E}_{\mathrm{y}}=-\frac{\mathrm{ab}}{\pi^{2}} \sum_{\mathrm{P}=1}^{\mathrm{M}} \mathrm{E}_{\mathrm{p}} \int_{-\infty}^{\infty} \int_{\infty}^{\infty} \operatorname{sinc}\left(\mathrm{k}_{\mathrm{y}} \mathrm{b}\right)$

$$
\frac{\left\{\begin{array}{l}
j \sin \left(\mathrm{k}_{\mathrm{x}} \mathrm{a}\right) \mathrm{p} \text { even } \\
\cos \left(\mathrm{k}_{\mathrm{x}} \mathrm{a}\right) \mathrm{p} \text { odd }
\end{array}\right\}}{\frac{\mathrm{p} \pi}{2}\left\{1-\left(\frac{2 \mathrm{ak}_{\mathrm{x}}}{\mathrm{p} \pi}\right)^{2}\right\}}
$$




$$
\begin{aligned}
& e^{j\left(k_{x} x_{1}+k_{y} y_{1}-k_{z} z_{1}\right)} d k_{x} d k_{y}, \\
& E_{z}=-\frac{a b}{\pi^{2}} \sum_{P=1}^{M} E_{p} \int_{-\infty}^{\infty} \int_{\infty}^{\infty} \frac{k_{y}}{k_{z}} \operatorname{sinc}\left(k_{y} b\right) \\
& \frac{\left\{\begin{array}{c}
j \sin \left(k_{x} a\right) p \text { even } \\
\cos \left(k_{x} a\right) p \text { odd }
\end{array}\right\}}{\frac{p \pi}{2}\left\{1-\left(\frac{2 a k_{x}}{p \pi}\right)^{2}\right\}} \\
& e^{j\left(k_{x} x_{1}+k_{y} y_{1}-k_{z} z_{1}\right)} d_{k_{x}} d k_{y},
\end{aligned}
$$

Where $\mathrm{E}_{\mathrm{p}}=$ basis function of the aperture radiator. Internally dispersed magnetic fields at the transmitter are obtained by using a modal expansion method [8]:

$$
\begin{aligned}
& \mathrm{H}_{\mathrm{x}}^{\mathrm{int} 1}=\sum_{\mathrm{P}=1}^{\mathrm{M}} \mathrm{E}_{\mathrm{p}}^{1} \mathrm{Y}_{\mathrm{p} 0}^{\mathrm{e}} \sin \left\{\frac{\mathrm{m} \pi}{2 \mathrm{a}}(\mathrm{x}+\mathrm{a})\right\}, \\
& \mathrm{H}_{\mathrm{x}}^{\mathrm{int} 2}=\sum_{\mathrm{P}=1}^{\mathrm{M}} \mathrm{E}_{\mathrm{p}}^{2} \mathrm{Y}_{\mathrm{p} 0}^{\mathrm{e}} \sin \left\{\frac{\mathrm{m} \pi}{2 \mathrm{a}}(\mathrm{x}+\mathrm{a})\right\},
\end{aligned}
$$

Where $\mathrm{E}_{\mathrm{p}}^{1}$ and $\mathrm{E}_{\mathrm{p}}^{2}$ are basis functions at the transmitting apertures, $\mathrm{m}=$ Number of half waves in $\mathrm{x}$-direction. At any inspection point on the poles, $\mathrm{x}$ and $\mathrm{y}$ component of the electric field dispersed by the induced current on the poles of the sensor are given by [10]:

$$
\begin{aligned}
& E_{y}^{S}=\frac{\lambda \sqrt{\frac{\mu}{\varepsilon}}}{8 \pi^{2} j} \int_{-l / 2}^{l / 2} I_{y p} \frac{e^{-j k R_{1}}}{R_{1}^{5}}\left[\left(1+j R_{1}\right)\left(2 R_{1}^{2}-3 a_{w}^{2}\right)+k^{2} a_{w}^{2} R_{1}^{2}\right] d y^{\prime}, \\
& E_{x}^{S}=\frac{\lambda \sqrt{\frac{\mu}{\varepsilon}}}{8 \pi^{2} j} \int_{-l / 2}^{l / 2} I_{x p} \frac{e^{-j k R_{2}}}{R_{2}^{5}}\left[\left(1+j k R_{2}\right)\left(2 R_{2}^{2}-3 a_{w}^{2}\right)+k^{2} a_{w}^{2} R_{2}^{2}\right] d x^{\prime},
\end{aligned}
$$

Where $\mathrm{R}_{1}=\sqrt{\mathrm{a}_{\mathrm{w}}^{2}+\left(\mathrm{y}-\mathrm{y}^{\prime}\right)^{2}}, \mathrm{R}_{2}=\sqrt{\mathrm{a}_{\mathrm{w}}^{2}+\left(\mathrm{x}-\mathrm{x}^{\prime}\right)^{2}}, 1, \mathrm{a}_{\mathrm{w}}=$ length and radius of the dipole, $\mathrm{x}, \mathrm{y}=$ any points of observation along the $\mathrm{x}$ and $\mathrm{y}$ direction in space, $\mathrm{x}^{\prime}, \mathrm{y}^{\prime}=$ points on the cross-pole and co-pole along $\mathrm{x}$ and $\mathrm{y}$-directions.

The electric field of the co and the cross pole due to mutual coupling effect is respectively given by [10]:

$\mathrm{E}_{\mathrm{y}}^{\mathrm{mx}}=\frac{\lambda \sqrt{\frac{\mu}{\varepsilon}}}{16 \pi^{3} \mathrm{j}}$

$\int_{x^{\prime}=-\frac{l}{2}}^{\frac{l}{2}} \int_{\phi^{\prime}=0}^{2 \pi} I_{x p} \frac{e^{-j k R_{3}}\left(x-x^{\prime}\right)\left(y-y^{\prime}\right)\left(3+3 j k R_{3}-k^{2} R_{3}^{2}\right)}{R_{3}^{5}}$

$\mathrm{dx}^{\prime} \mathrm{d} \phi^{\prime}$

$E_{x}^{m y}=\frac{\lambda \sqrt{\frac{\mu}{\varepsilon}}}{16 \pi^{3} j} \int_{y^{\prime}=-l / 2}^{l / 2} \int_{\phi^{\prime}=0}^{2 \pi} I_{y p} \frac{e^{-j k R_{4}}\left(x-x^{\prime}\right)\left(y-y^{\prime}\right)\left(3+3 j k R_{4}-k^{2} R_{4}^{2}\right)}{R_{4}^{5}}$

dy d $\phi^{\prime}$

Where $R_{3}=\sqrt{\rho^{2}+a_{w}^{2}-2 \rho a_{w} \cos \phi^{\prime}\left(x-x^{\prime}\right)^{2}}, \rho=$ radial distance to the observation point, $\phi^{\prime}=$ Azimuth angle at the dipole, $R_{4}=\sqrt{\rho^{2}+a_{w}^{2}-2 \rho a_{w} \cos \phi^{\prime}\left(y-y^{\prime}\right)^{2}}$.

\section{Imposition of boundary condition}

The boundary conditions at the transmitter openings are tangential magnetic field components both inside and outside the waveguides are equal and are given by

$2 H_{x}^{\text {inc1 }}+H_{x}^{\text {int } 1}=H_{x}^{\text {ext1 }}+H_{x}^{\text {ext12 }}+H_{x}^{\text {extco }}+H_{x}^{\text {extcr }}$

$2 H_{x}^{\text {inc2 }}+H_{x}^{\text {int2 }}=H_{x}^{\text {ext2 }}+H_{x}^{\text {ext21 }} H_{x}^{\text {extco }}+H_{x}^{\text {extcr }}$,

Where $H_{x}^{\text {inc1 }}, H_{x}^{\text {inc2 }}=$ Incident magnetic field in apertures 1 and $2, H_{x}^{\text {int } 1}, H_{x}^{\text {int2 }}=$ Internally scattered magnetic field inside the apertures 1 and $2, H_{x}^{\text {ext } 1}, H_{x}^{\text {ext2 }}=$ Externally radiated magnetic field from apertures 1 and $2, H_{x}^{\text {ext } 12}, H_{x}^{\text {ext } 21}=$ Externally radiated magnetic field from aperture 1 to aperture 2 and vice versa, $H_{x}^{\text {extco }}, H_{x}^{\text {extcr }}=$ externally radiated magnetic field from apertures to co-pole and cross pole respectively.

The boundary conditions on the axis of the sensor is total tangential components of the electric field is zero.

$$
E_{y}^{i n c}+E_{y}^{m x}+E_{y}^{s c a t}=0,(19) E_{x}^{i n c}+E_{x}^{m y}+E_{x}^{s c a t}=0
$$


Where $E_{y}^{i n c}, E_{x}^{i n c}=$ incident electric fields on co-pole and cross-pole respectively, $E_{y}^{m x}, E_{x}^{m y}=$ mutually coupled electric fields between co-pole to cross-pole and vice-versa, $E_{y}^{\text {scat }}, E_{x}^{\text {scat }}=$ scattered electric fields from co-pole and cross-pole respectively.

The field moment and weighting functions are well-defined as follows [8] and [10]:

$\left\langle H, w_{q}^{1}\right\rangle=\iint_{\text {surface }} H, w_{q}^{1} d x d y$,

$\left\langle E, w_{q}^{2}\right\rangle=\iint_{\text {surface }} E, w_{q}^{2} d s$,

Where $w_{q}^{1}, w_{q}^{2}=$ global and Dirac delta weighting functions of the transmitter opening and on the pole surface respectively and these are given by,

$w_{q}^{1}=\left\{\begin{array}{c}\sin \left\{\frac{q \pi}{2 a}(x+a)\right\},\left\{\begin{array}{l}-a \leq x \leq a \\ -b \leq y \leq b\end{array}\right. \\ 0, \text { elsewhere }\end{array}\right.$

$w_{q}^{2}=\left[\delta\left(h-h_{q}\right)\right],(24)$

Where $h=$ point with respect to some reference (origin), $h_{q}=$ position at which the boundary condition is forced on the poles.

Using the boundary conditions given by Equations (17), (18), (19) and (20) and definition in Equations (21) and (22), converting these linear equations in matrix form by taking moments.

The moments of the incident and dispersed fields are given by

$\left[\begin{array}{l}E_{p}^{1} \\ E_{p}^{2} \\ y_{p} \\ x_{p}\end{array}\right]=\left[\begin{array}{rlll}{\left[L^{\text {ext } 1}\right]-\left[L^{\text {int } 1}\right]} & {\left[L^{\text {ext } 2}\right]-\left[L^{\text {int } 2}\right]} & {\left[L^{\text {extco }}\right]} & {\left[L^{\text {extcr }}\right]} \\ {\left[L^{\text {ext } 2}\right]-\left[L^{\text {int } 2}\right]} & {\left[L^{\text {ext } 1}\right]-\left[L^{\text {int } 1}\right]} & {\left[L^{\text {extco }}\right]} & {\left[L^{\text {extcr }}\right]} \\ {\left[L^{\text {coinc } 1}\right]} & {\left[L^{\text {coinc }}\right]} & {\left[L^{\text {coscat }}\right]} & {\left[L^{\text {ymx }}\right]} \\ {\left[L^{\text {coinc } 2}\right]} & {\left[L^{\text {coinc } 1}\right]} & {\left[L^{\text {crscat }}\right]} & {\left[L^{\text {ymx }}\right]}\end{array}\right]^{-1}$

$\left[\begin{array}{c}2[L]^{i n c 1} \\ 2[L]^{i n c 2} \\ {[0]} \\ {[0]}\end{array}\right]$

From the above equation co-efficient of global basis and Dirac delta functions are simultaneously determined. From these basis functions, waveguide transmitters reflection co-efficient, admittance, susceptance and sensor voltage are determined.

\section{Waveguide reflection coefficient}

Reflection coefficient $\Gamma$ can then be stated as

$\Gamma=\frac{E_{y}^{S}+E_{y}^{1}+E_{y}^{2}}{E_{y}^{\text {inc }}}$

Where $E_{y}^{S}, E_{y}^{i n c}=$ reflected and incident electric field, respectively, and given by,

$E_{y}^{S}=-\cos \left(\frac{\pi x}{2 a}\right), E_{y}^{i n c}=\cos \left(\frac{\pi x}{2 a}\right)$,

$E_{y}^{1}, E_{y}^{2}=$ fields of the dominant mode scattered by the apertures 1 and 2 into the waveguide are derived from equations (7) and (8).

The following relation gives the admittance:

$Y=\frac{1-\Gamma}{1+\Gamma}$, Where $Y=G \pm j B$,

$G=$ Conductance, $B=$ Suceptance

\section{Numerical results}

The opening in an unbounded ground plate fed by a standard X-band WR-90 rectangular waveguide is used as a transmitting antenna and thin, finite length, perfectly conducting crossed dipole of length $0.47 \lambda$ and radius of $0.005 \lambda$, used as a near field sensor. As it is not possible to connect lumped load of $50 \Omega$ at the center point of the crossed dipole, hence it is connected at the point which is one pulse basis function away from the center of each pole as shown in Figure 1. In order to calculate the coefficients of the basis functions, the program is written in MATLAB 12.

A computation has been carried out to sample the co-pole $\left(E_{y}\right)$ voltage and relative electric field (without probe) in the scanning plane at $10 \mathrm{GHz}$ and comparison has been done between these two patterns. No error is imposed at the midpoint of the scan plane. These graphs are shown in Figure. 2 and Figure. 3 in the scanning plane at $x=0$, and $z=1.0 \lambda$ and $5.0 \lambda$ respectively. The error in the normalized voltage waveform shape with respect to the normalized electric field (without probe) pattern is calculated. 


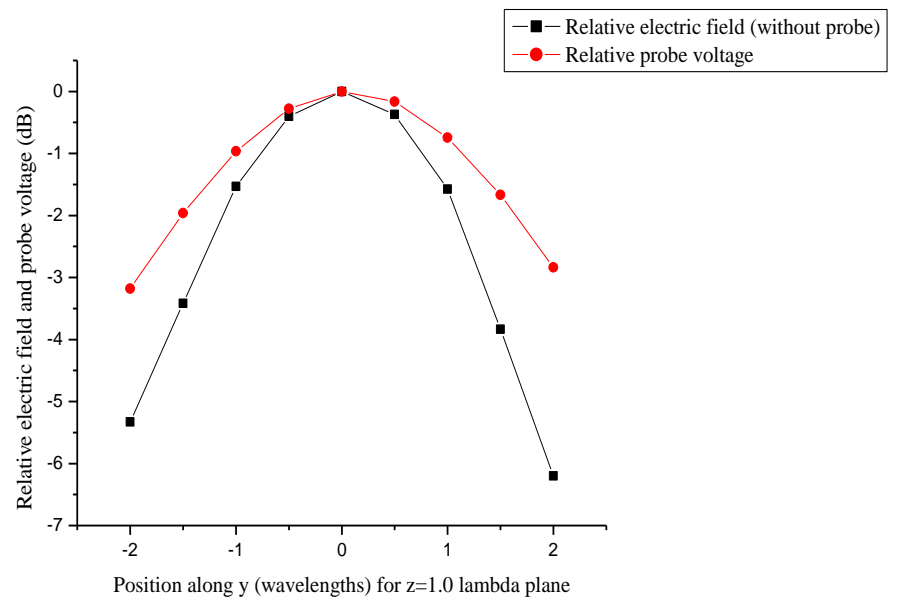

Fig. 2: Relative Sampled Electric Field (Without Probe) and Co-Pole Voltage in $X-Y$ Plane at $X=0, Z=1.0 \lambda$ at 10ghz.

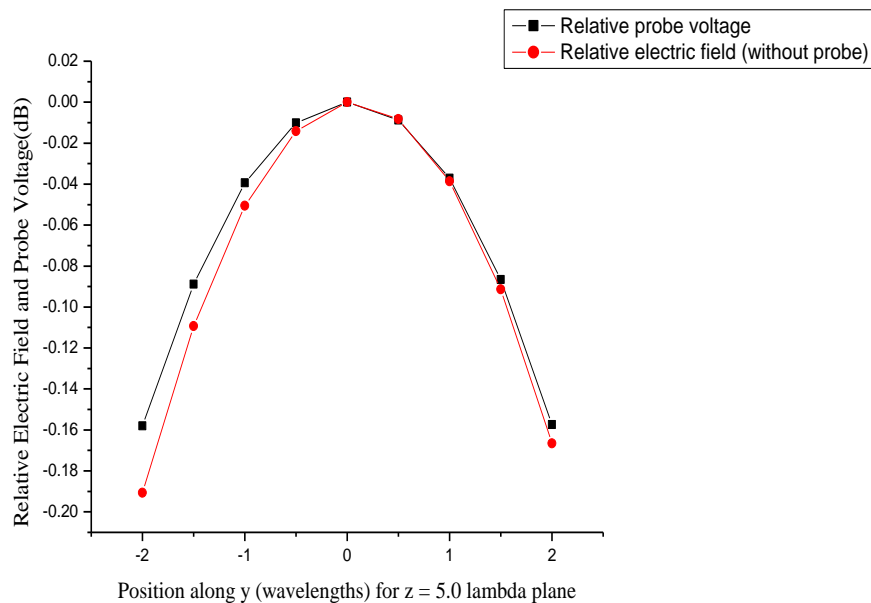

Fig. 3: Relative Sampled Electric Field (Without Probe) and Co-Pole Voltage in X-Y Plane at $X=0, Z=5.0 \Lambda$ at $10 \mathrm{ghz}$.

The radiators reflection coefficient in the sight of the near field measuring probe with each pole length $\mathrm{L}=1.4 \mathrm{~cm}$ and radius $\mathrm{a}_{\mathrm{w}}=0.015 \mathrm{~cm}$, (designed at $10 \mathrm{GHz}$ ) at $\mathrm{x}=0, \mathrm{y}=0$ and $\mathrm{z}=1.5 \mathrm{~cm}, 3.0 \mathrm{~cm}, 5.0 \mathrm{~cm}, 8.0 \mathrm{~cm}$ and in the absence of the measuring probe, over 8 to $12 \mathrm{GHz}$. These results are compared to the experimentally measured results and are shown in Figure. 5. The calculation for radiation conductance $(\mathrm{G})$ and susceptance (B) have been carried out, at $\mathrm{x}=0, \mathrm{y}=0$ and $\mathrm{z}=1.5 \mathrm{~cm}, 3.0 \mathrm{~cm}, 5.0 \mathrm{~cm}, 8.0 \mathrm{~cm}$ and in the absence of the sensor over 8 to $12 \mathrm{GHz}$, and is shown in Figure.6 and Figure.7.

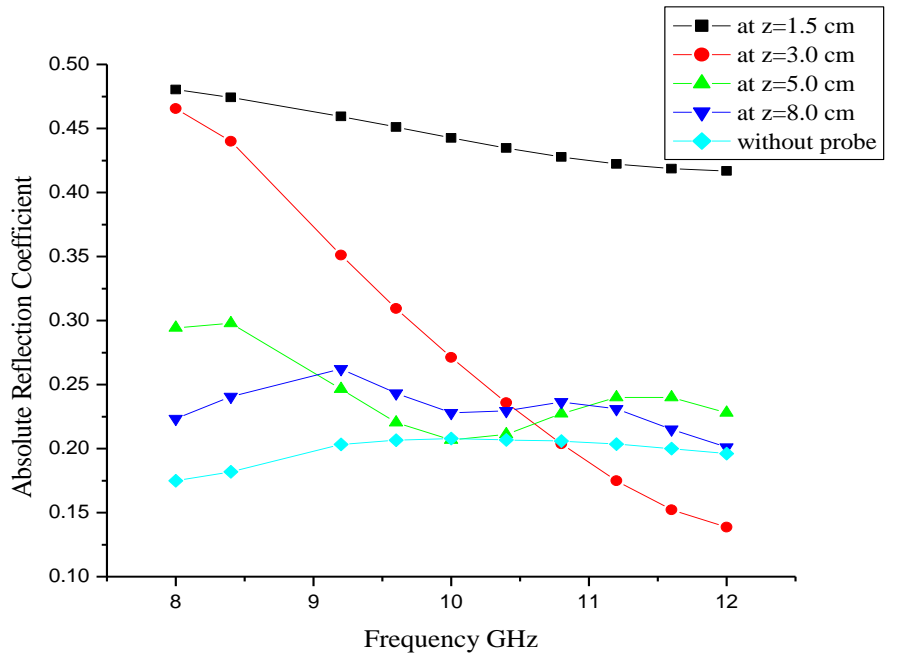

Fig. 4: Absolute Reflection Coefficient for the Radiator Array in the Presence of the Probe at $X=0, Y=0$ and $Z=1.5 \mathrm{~cm} / 3.0 \mathrm{~cm} / 5.0 \mathrm{~cm} / 8.0 \mathrm{~cm}$, and in the Absence of the Probe Over 8 to $12 \mathrm{Ghz}$. 


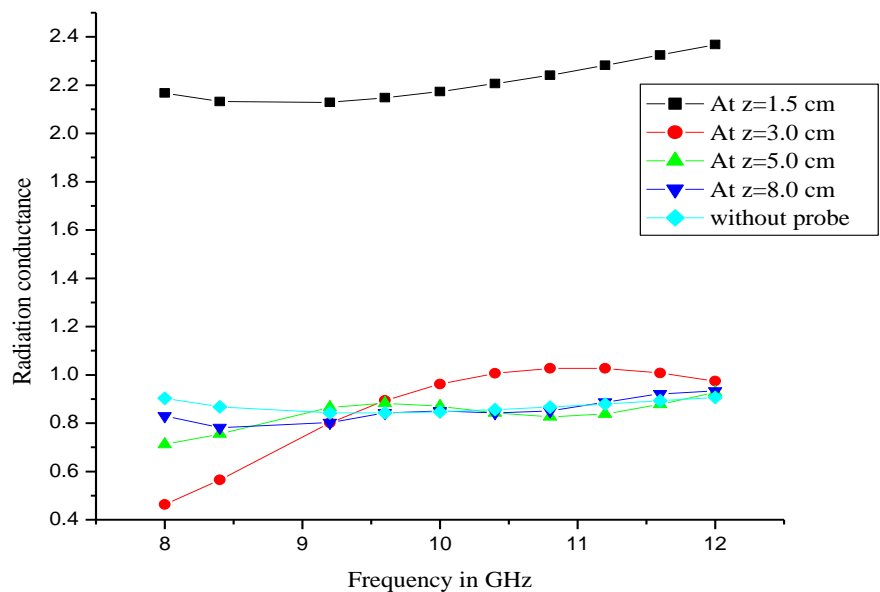

Fig. 5: Radiation Conductance for Radiator Array in the Presence of the Probe at $X=0, Y=0$ and $Z=1.5 \mathrm{~cm} / 3.0 \mathrm{~cm} / 5.0 \mathrm{~cm} / 8.0 \mathrm{~cm}$ and in the $A b s e n c e$ of The Probe, Over 8 to $12 \mathrm{Ghz}$.

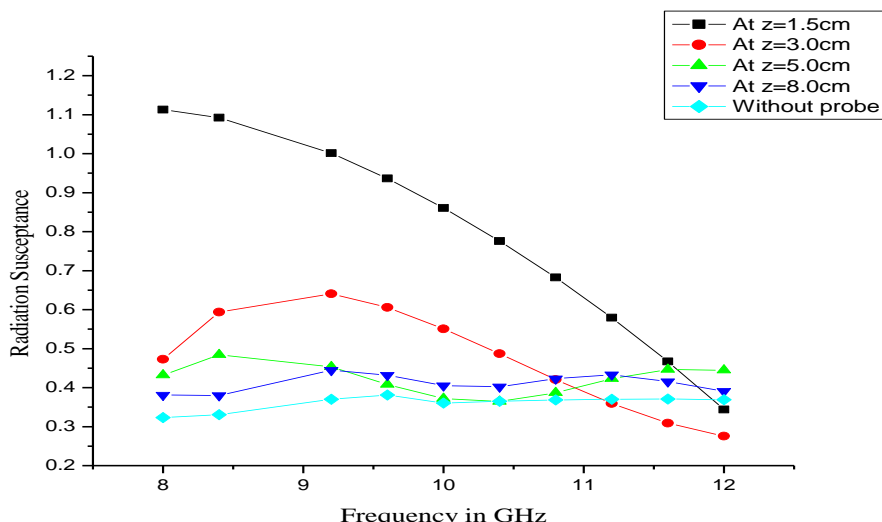

Fig. 6: Radiation Susceptance For Radiator Array in the Presence of the Probe at $X=0$, $Y=0$ and $Z=1.5 \mathrm{~cm} / 3.0 \mathrm{~cm} / 5.0 \mathrm{~cm} / 8.0 \mathrm{~cm}$ and in the Absence of The Probe, Over 8 to $12 \mathrm{Ghz}$.

\section{Conclusion}

The crossed dipole is used as a near-field measuring probe. The electric field at the center of the scan plane is larger and decreases at away from the center. The junction point of the probe is taken as a reference and it is not exactly at the center of the poles, but it is one pulse basis function away from the center, due to this maximum co-pole voltage slightly deviates from the center of the scan plane. However, because of the multiple reflections between the radiator and probe, and scattering properties of the conducting probe, near-field values and radiating characteristics of the waveguide are altered. The error in the relative sampled voltage pattern of measuring probe with respect to the electric field (without probe) is shown in Figure. 2 and Figure. 3. As the measuring element approaches the radiator, the error produced due to induction by the probe is increased. As the probe moves away from the radiator, error induced will be less, due to the smaller effect of multiple reflection and scattering of the probe.

In the presence of the near field measuring probe, the radiator reflection coefficient, admittance and susceptance have been changed and presented in Figure. 4, Figure. 5 and Figure. 6. When the distance between the source and the measuring element is lesser, the deviations of the reflection coefficient, admittance and susceptance are larger with respect to the corresponding values when there is no measuring element in the near field. As the separation increases, due to the decreased effect of multiple reflection and scattering of the probe, the deviation is smaller, and for larger separation, these approaches the values obtained, when there is no measuring element in the near field. However, due to multiple reflections between the transmitter and measuring probe, near-field values and radiation characteristics of the radiator are changed.

\section{References}

[1] G. V. Borgiotti, "Fourier transform method in aperture antenna problems," Alta Frequenza, vol. 32, Nov. 1963.

[2] R. J. Mailloux, "Radiation and near-field coupling between two collinear open-ended waveguides," IEEE Trans. on Antennas and Propagation, vol. AP-17, Jan. 1969. https://doi.org/10.1109/TAP.1969.1139450.

[3] G. V. Borgiotti, "A novel expression for the mutual admittance of planar radiating elements," IEEE Trans. on Antennas and Propagation, vol. AP16, pp. 329-333, May 1968 https://doi.org/10.1109/TAP.1968.1139185.

[4] A. D. Yaghjian, "Efficient computation of antenna coupling and fields within the near-field region," IEEE Trans. on Antenna and Propagation, vol. AP-30, No. 1, Jan. 1982. https://doi.org/10.1109/TAP.1982.1142752.

[5] H. Baudrand, Jun-Wu Tao and J. Atechian "Study of radiating properties of open-ended, rectangular waveguides," IEEE Trans. on Antennas and Propagation, vol. AP-36, pp. 1071-1077, Aug. 1988. https://doi.org/10.1109/8.7219.

[6] D.G. Bodnar and D.T. Paris, "New variational principle in electromagnetics," IEEE Trans. Antennas Propagation, vol. AP-19.216-223, Mar.1970. https://doi.org/10.1109/TAP.1970.1139642.

[7] R.H. MacPhie and A.I. Zaghloul, "Radiation from a rectangular waveguide with infinite flange: Exact solution by correlation matrix," IEEE Trans. Antennas Propagation, Vol. AP-28, pp 497- 503,1980. https://doi.org/10.1109/TAP.1980.1142376.

[8] Paramesha and A. Chakrabarty, "Waveguide as a Near Field Measuring Probe of the Two Element Array Radiator." Progress In Electromagnetics Research B, Vol.7, pp 245-255, 2008. https://doi.org/10.2528/PIERB08032401. 
[9] Paramesha and A. Chakrabarty, "Near-Field Distraction due to the Probe," International conference on Antenna and Technology, Space Application Centre, ISRO, Ahmadabad, Feb. 2005.

[10] Paramesha and A. Chakrabarty, "Near-Field Measurement Using Crossed Dipole," International Radar Symposium India, IRSI-05, Bangalore, Dec. 2005.

[11] J.H. Kim, B. Enkhbayar, J.H. Bang, and B.C. Ahn, "New formulas for the Reflection Coefficient of an Open-ended Rectangular Waveguide Radiating into air Including the Effect of Wall Thickness or Flange," Progress in Electromagnetics Research M, Vol. 12, 143-153, 2010. https://doi.org/10.2528/PIERM10033104. 